\title{
Shortening subtrochanteric osteotomy of the femur in total hip arthroplasty in patients with congenital hip dislocation
}

\author{
L.S. Shnaider, O.I. Golenkov, E.U. Turgunov, M.V. Efimenko, M.A. Stepankova, V.V. Pavlov
}

Novosibirsk Research Institute Of Traumatology And Orthopaedics N.a. Ya.l. Tsivyan, Novosibirsk, Russian Federation

Congenital hip dislocation in adults (Crowe type IV) is an indication for hip replacement. Shortening subtrochanteric osteotomy of the femur reduces the risk of vascular and neurological complications and corrects the antetorsion of the proximal femur. Purpose To demonstrate our experience with the use of the method of shortening osteotomy of the femur in hip joint replacement for congenital hip dislocation with an assessment of postoperative complications. Materials and methods 64 hip arthroplasties were performed using corrective osteotomy. The follow-up period was from 6 months to 8 years. We evaluated pain and functional status according to VAS and Harris Hip Score. Results Pain according to VAS in the hip was $3.4 \pm 1.1$ and $2.1 \pm 2.2$ in the lumbar spine. HHS was $55.6 \pm 17.2$ points. Pain relief in the lumbar spine might be associated with changes in the lateral radiographs of the lumbar spine. In the postoperative period, pelvic anteversion returned to normal, as did the other angles of the lumbar-pelvic balance. Conclusions Hip joint arthroplasty for Crowe type IV hip dysplasia is an operation of high complexity and is associated with a high risk of complications (19.7 \%).

Keywords: osteotomy, congenital hip dislocation, Crowe IV, hip dysplasia

\section{INTRODUCTION}

"Congenital Dislocation of the Hip (CDH)" is interpreted as dysplastic coxarthrosis by domestic literature due to congenital dislocation of the femur while and "Developmental Dysplasia of the Hip (DDH)" as dysplastic coxarthrosis with varying degrees of cranial displacement of the femur relative to the pelvis, which is specified with various classifications such as Crowe [1], Hartofilakidis [2], Eftekhar [3]. These classifications refer the extreme grade of femoral head displacement to congenital hip dislocation. Subsequently, G. Hartofilakidis in 2004 identified two subtypes in the class of high dislocation to clarify the relationship between the head of the femur and the false cavity on the wing of the ilium, supportive (Crowe IV, Hartofilakidis C1) or non-supportive (Crowe IV, Hartofilakidis C2);

In bilateral dysplasia with its different severity, the relative and anatomical length of the lower extremities will differ. In non-supportive dislocation (Hartofilakidis C2), the head of the femur is not under the physiological load of the body weight, and does not result in physiological curves of the femur, which leads to its maximum anatomical length. If there is a contralateral normal hip joint $(\mathrm{HJ})$ or a supportive dislocation, then due to the load of the body weight, physiological varus and antecurvatum is formed leading to a decrease in the length.

The choice for surgical treatment is hip arthroplasty, which presents the greatest technical challenge for the orthopedic surgeon. The reason is the complexity of preoperative planning, technical difficulties of the operation performance itself associated with previous interventions, presence of scars, the need to bring down the femoral head, etc.; greater trauma and duration of the operation, increased blood loss compared to standard arthroplasty; a significant number of local intra- and postoperative complications.

The acetabulum in patients with high hip dislocation is characterized by bone tissue deficit in the area of anterolateral and superior walls to obtain sufficient bone coverage of the cup, by underdevelopment and deformation of the bone components of the joint, and their mutual displacement. The proximal femur is characterized by hypoplasia with a narrow intramedullary canal and excessive antetorsion. In addition, the muscles and soft tissues of the thigh are shortened and the capsule is thickened. The sciatic nerve is shortened and may be injured if the limb needs to be lengthened by more than $4 \mathrm{~cm}$. It is reported that sciatic nerve palsy occurs in 0.8 to $13 \%$ of patients with hip dysplasia who undergo hip arthroplasty [4].

Excessive mobilization of the proximal femur during the operation might cause failure of the gluteal muscles, and pronounced tension of the soft tissues is fraught with traction damage to the femoral and sciatic nerves.

To solve this problem, orthopedic surgeons refer to the work of Klisic and Jankovic [5] who for the first time in the era of widespread use of antibiotics performed shortening femoral osteotomies in children to treat high dislocations of the hip. Sponseller [6] was the first to use femoral osteotomy in hip

Shnaider L.S., Golenkov O.I., Turgunov E.U., Efimenko M.V., Stepankova M.A., Pavlov V.V. Shortening subtrochanteric osteotomy of the femur in total hip arthroplasty in patients with congenital hip dislocation. Genij Ortopedii, 2020, vol. 26, no 3, pp. 340-346. DOI 10.18019/1028-4427-2020-26-3-340-346 
arthroplasty. Developing the femur osteotomy for hip arthroplasty, Cabenala [7] outlined the requirements for this osteotomy. It should be subtrochanteric, with mandatory antetorsion of the proximal femur fragment and distal fixation of the cementless stem to the distal femur fragment. Using these guidelines, Park et al. [8] reported 24 cases of arthroplasty with subtrochanteric osteotomy without neurological complications. Krych et al. in 2009 [9] reported 5 -year observations with a final HHS of 89 points.

As for the problem of restoring the length of the lower limb and planning the resection of the femur during the performance of a shortening subtrochanteric osteotomy, it remains relevant. Therefore, planning the operation, it is necessary to take into account the following: the nature of high dislocation, supportive (Crowe IV, Hartofilakidis C1) or non-supportive (Crowe IV, Hartofilakidis C2); unilateral or bilateral involvement; previous operations; knee joint contracture on the side of dislocation.

Thus, the shortening subtrochanteric osteotomy of the femur enables to:

1) preserve the integrity of the proximal femur fragment;

2) move the distal fragment of the femur without tension;

3) minimize the risk of damage to the sciatic and femoral nerves;

4) maintain the functional (projectional) length of the lower limb.

The aim of this work was to demonstrate our experience in the application of the method of shortening subtrochanteric osteotomy of the femur in hip arthroplasty for congenital hip dislocation, as well as to assess postoperative complications of this intervention. Based on the above, we hope that the presentation of our methodology will be a demand.

\section{MATERIAL AND METHODS}

In the period from 2008 to September 2018, 47 patients were operated, including five men and 42 women, whose average age was $42.4 \pm 12.6$ years. Inclusion criteria were severe pain in the hip and significant difficulty by walking and performing daily activities, supported by radiographic diagnosis of unilateral or bilateral Crowe type IV hip dysplasia. Twenty-six patients were operated for unilateral hip dysplasia of grade IV according to Crowe (D1). Twenty-one patients with bilateral Crowe IV dysplasia (D2) underwent operations; 17 of them were operated on both sides, and four refused to undergo contralateral arthroplasty after stage 1 as three of them had complications. Thus, the total number of hip arthroplasties performed was 64 .

The follow-up period ranged from 6 months to 8 years.

The method of hip arthroplasty along with corrective osteotomy (patent 2533971 RF: IPC A61B17 / 56, A61B6 / 00; November 27, 2014) were used in all the patients.

The volume of bone resection in the femur was analyzed, the initial and final length of the femurs and lower extremities, the timing of consolidation, the number and nature of complications specific to this technique were assessed.

In the course of the study, pain was assessed using the VAS scale in the hip joint and spine, and the functional status was assessed using the Harris scale. Sacral slope (SS), lumbar lordosis (GLL), and pelvic anteversion (PT) were assessed on lateral radiographs of the lumbar spine (LS) with hip capture.

A shortening subtrochanteric osteotomy of the femur was performed for Crowe IV hip dysplasia for bringing the femur down, avoiding nerve damage, preserving the attachment points of the muscles in the region of the greater trochanter. It is very important for biomechanics of the joint.

Preoperative planning includes a direct pelvic radiograph and a lateral radiograph of the hip joint. We find the rotation center of the femoral head, then the "Ranovat" triangle is drawn to determine the true center of rotation. Then we measure the distance from the femoral head to the true center of rotation to obtain the maximum amount of the femur resection. Finally, the magnitude of femur resection is determined intraoperatively in order to maximally compensate for the length of the limb, especially in unilateral dislocation.

\section{Surgical technique}

Anterolateral approach was used. Upon access to the proximal femur, the greater trochanter was identified, the rudimentary head of the femur was palpated, and the stretched capsule of the joint was exposed and cut. It was incised over the palpable head, and then the latter was dislocated and resected along the intertrochanteric line. Next, myolysis of the gluteus medius muscle was performed until it was fixed to the wing of the ilium and the tendon of the iliopsoas muscle was cut off from the lesser trochanter. After that, traction of the lower extremity was carried out and the osteotomy of the femur was marked with a transverse line at a distance of $1 \mathrm{~cm}$ distal to the lesser trochanter. As a rule, it was $5-6 \mathrm{~cm}$ from the apex of the greater trochanter and corresponded to the centre of rotation of the acetabulum (Fig. 1).

Prior to transverse osteotomy of the femur, a superficial longitudinal sawing of the bonewas produced with an oscillator saw along the axis of the femur to maintain rotational orientation for its restoration. After the osteotomy of the femur, its proximal fragment with preserved gluteus medius muscle was retracted upward 
with additional mobilization along its posterior surface, and the distal fragment was retracted posteriorly. Thus, a convenient approach to the true acetabulum was created. The capsule and scar tissue were excised. Next, retractors were installed behind the anterior, anteriorinferior and posterior edges of the acetabulum. The acetabulum was formed using cutters, starting with a $40 \mathrm{~mm}$ cutter. The cavity, as a rule, was processed in the reverse mode in order to impress the bone tissue, thereby minimizing its loss. Then, the selected size and position of the acetabular component were tested with test components inserted at an angle to the frontal plane of $30-40^{\circ}$ and $10-15^{\circ}$ to the sagittal plane of the pelvic component, followed by implantation of the pelvic component selected. In $63.5 \%$ of cases, the acetabular component was additionally fixed with 2 screws. It depended on the degree of under-coverage of the pelvic component and the surgeon's subjective feelings.

Treatment of the femur began with manual traction of the involved lower limb in the distal direction, paying attention to the danger of damage to the sciatic nerve, and proceeded to determine the second level of resection of the femur according to the preoperative planning and focusing on the mobility of the femur. While producing manual traction, the proximal femur fragment was displaced as much as possible downwards along the axis of the distal fragment. At the level of its maximum displacement, a transverse mark was done on the distal fragment of the femur, which was a reference point for performing the second level of resection. The resected fragment of the femur (from 40 to $50 \mathrm{~mm}$ ) was removed, with obligatory mobilization along the linea aspera, since it is wide at this level and with a large number of vessels.

The next step was sequential processing of the distal and proximal femur fragments. First, the reamers were inserted into the distal fragment, so that the installed femoral component was immersed in the medullary canal by $2 / 3$. Having reached the appropriate immersion and the desired size, we proceeded to the next stage. An 8-mm drill was inserted retrograde along the axis of the proximal fragment and its exit in the projection of the piriform fossa was monitored. Marking the exit point in the area of the piriform fossa, through it, reamers were sequentially introduced into the proximal femur fragment, so that the reamer of the last size (selected when processing the distal fragment) protruded from the end of the proximal femur to a length of $2 / 3$ of the corresponding immersion into the distal section of the femur.

To prevent splitting of the proximal femur (in the absence of osteoporosis), a reamer was used one size larger and to the same immersion. Fragments were collected on a test femoral component corresponding to the size with restoration of the torsion of the proximal femur using a previously performed longitudinal cut or at $15^{\circ}$ anteriorly inclined. In addition, the cortical bone in the area of the Adams arch was cut with nippers for optimal contact of the surface of the femoral component with the bone. The femoral component was carefully hammered in in the distal fragment, pressing the proximal fragment to the latter. When creating a new position ( $15^{\circ}$ torsion anteriorly), a longitudinal cut was made, thus marking the achieved torsion of the proximal bone fragment with the matched femoral fragments. Later, by implanting the femoral component, we were guided by it. The shapes of the end ends as a result of the removal of an intermediate bone fragment, as a rule, are not adapted when juxtaposed, but it is not necessary to pay attention to this condition. The template of the femoral component with the S-head was reduced into the acetabulum. If the reduction was achieved without technical difficulties, and the clearnce suited us, we proceeded to the osteotomy of a "Russian lock" type (Fig. 2).
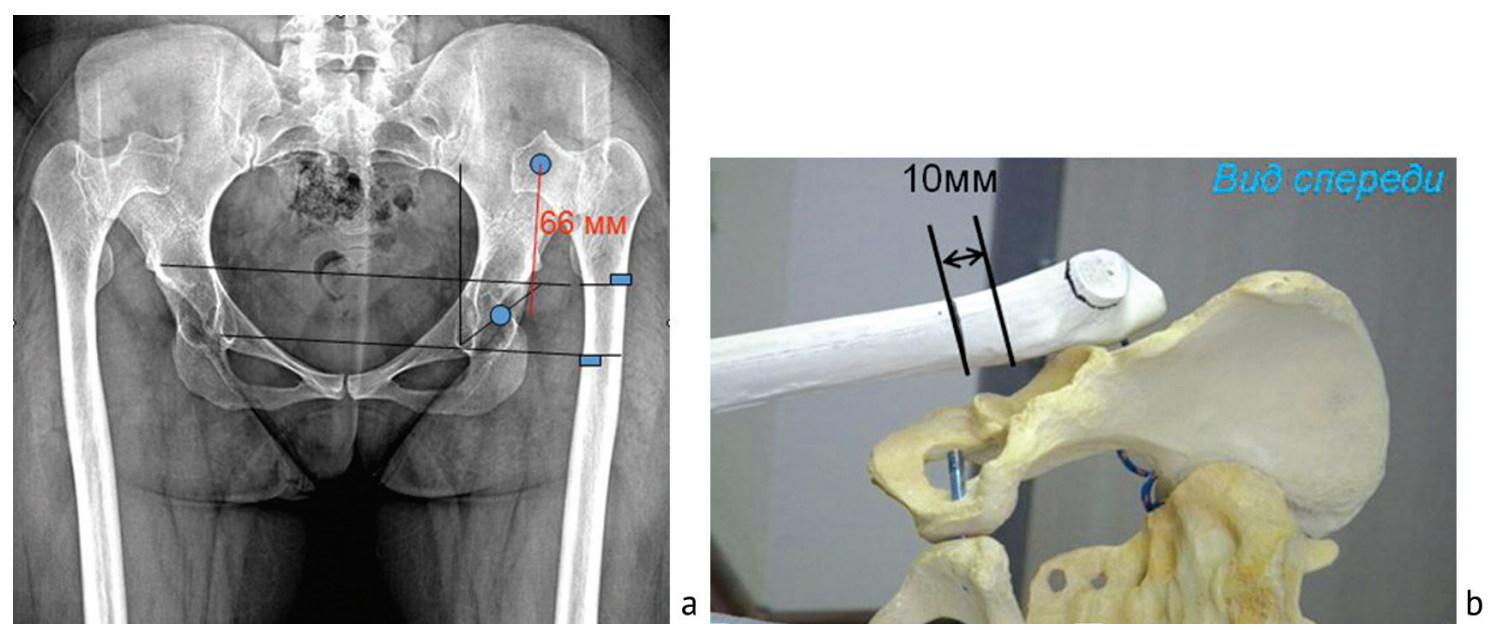

Fig. 1 (a) X-ray of the pelvis and hip joints in frontal projection: dysplastic coxarthrosis, Crowe type IV. Bilateral nonsupportive dislocation (Hartofilakidis C2). Planning femoral resection; (b) osteotomy zone in the femur model 

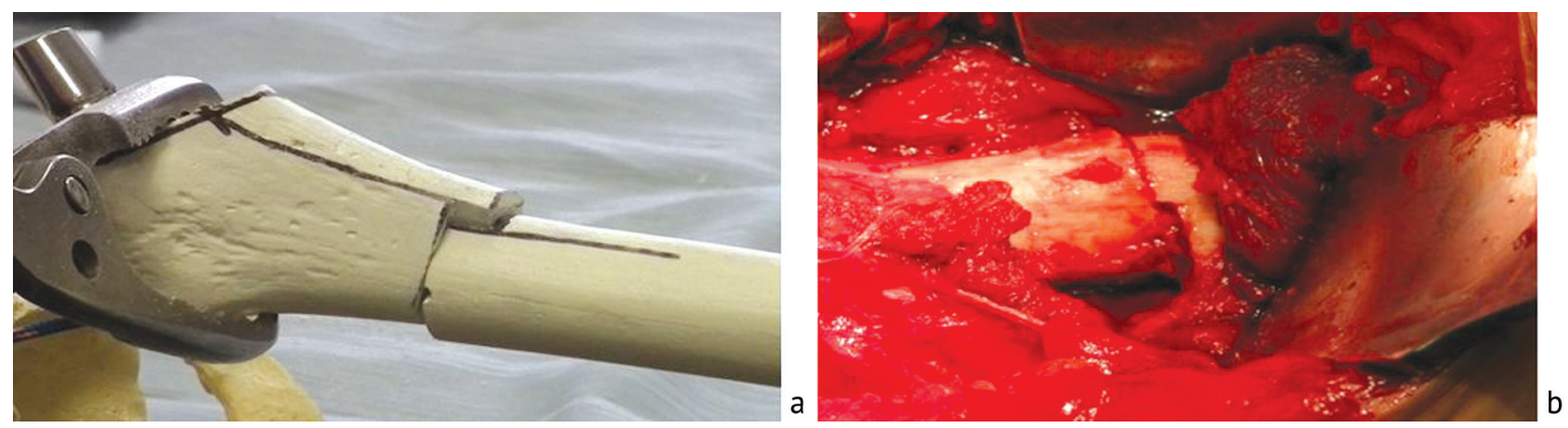

Fig. 2 Osteotomy produced on a model $(a)$ and its view in the wound $(b)$

If it was impossible to reduce the femoral component by traction of the limb, we proceeded as follows. In the position of traction, the distance between the centre of the head and the centre of the acetabulum was measured. The measured distance was transferred to the distal fragment of the femur for additional filing, adding 3.5 or $4 \mathrm{~mm}$ to form a "Russian lock"-type junction, which corresponded to the size of the head. The implementation of the connection of the "Russian lock" (Z-shaped osteotomy) with a difference in the size of the end surfaces of the fragments was carried out as follows. For convenience, the first stage was performed with longitudinal cuts of the ends by the amount of the size range of the heads, and then transverse cuts, retreating from the edge of the bone by the amount of the longitudinal cut. The first transverse cut was made on the most protruding part of the bone (it could be located on any fragment), and the second transverse cut was made on the less protruding opposite side of the corresponding opposite fragment. Then, a trial reduction was performed without the femoral component to assess the parallelism of the cuts and, if all circumstances were satisfactory, a test implantation of the femoral component was performed. Rotational instability was checked and, if absent, definitive implantation of the femoral component was performed.

If rotational instability was found, the test component was removed and the next a larger one was tried for retesting without additional processing of the distal fragment. Performing the described osteotomy provides good rotational stability of the femur fragments and does not require additional MOS of the osteotomy site. In order to prevent periprosthetic fractures in poor bone tissue condition or by cracking of the femur during its processing, cerclage sutures were applied close to the osteotomy area. Having achieved stability, the femoral component was implanted. Since 2012, we have used the femoral components of only a conical shape and of standard length. In our opinion, they are optimal for the dysplastic shape of the femoral canal. After the implantation, the femoral component was tested for dislocation; if necessary, the head was replaced with a longer one to eliminate the latter. The wound was drained and sutured in layers with an aseptic dressing. The condition after surgery is presented Figure 3.
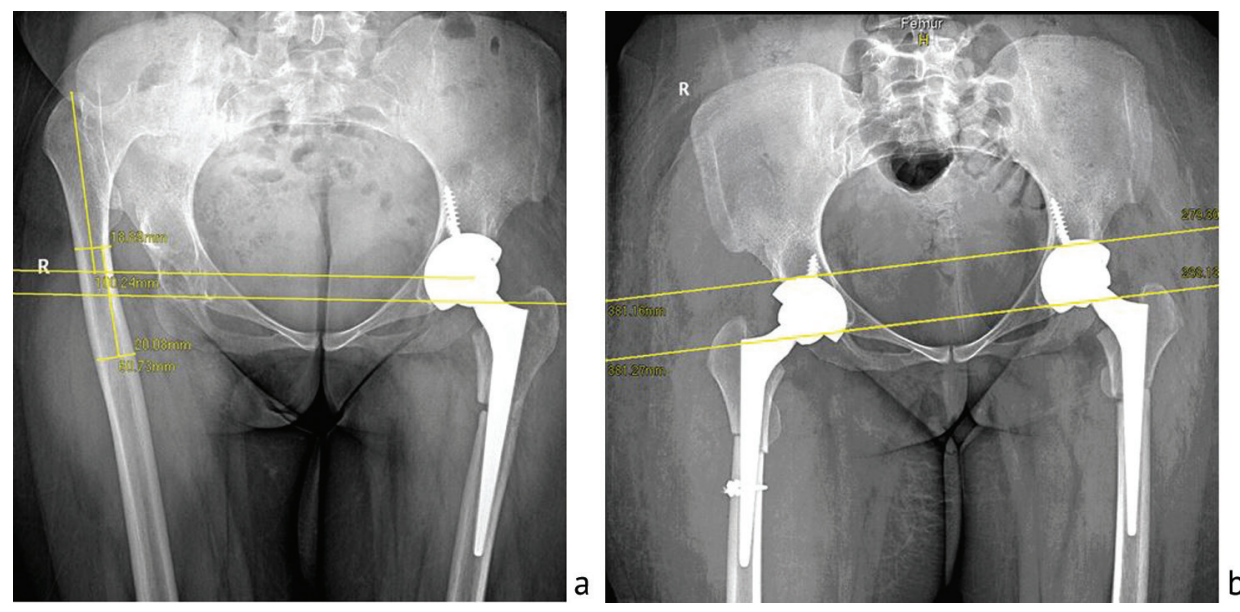

Fig. 3 Condition after total arthroplasty that includes shortening subtrochanteric osteotomy: (a) after the first stage of surgical treatment; (b) after the second stage of surgical treatment

\section{RESULTS}

The distance from the centre of rotation to the teardrop figure before surgery was $88 \pm 19.7 \mathrm{~mm}$ and $13.4 \pm 17.0 \mathrm{~mm}$ after it. We used the following sizes of the pelvic components: $42 \mathrm{~mm}$ in 2 cases, $44 \mathrm{~mm}$ in
45 cases, $46 \mathrm{~mm}$ in 14 cases, $48 \mathrm{~mm}$ in 4 cases, $50 \mathrm{~mm}$ in one case and $54 \mathrm{~mm}$ in one case. Additional fixation of the pelvic component with screws was required in 40 cases $(63.5 \%)$. Augments were installed for 
additional support of the pelvic component in two cases; bone plasty with the femoral head was performed in one case. Average offset after surgery was $34.3 \pm 5.3 \mathrm{~mm}$. It was necessary to strengthen the osteotomy fragments with cerclage in 18 (27.3\%) cases.

Table 1

Mean values by arthroplasty manipulations

\begin{tabular}{|l|l|}
\hline \multirow{3}{*}{ Shift of rotation centre } & Downwards 9.1 $\pm 4.21 \mathrm{~mm}(\mathrm{n}=59)$ \\
\cline { 2 - 2 } & Lateral 5.27 $\pm 2.52 \mathrm{~mm}(\mathrm{n}=31)$ \\
\cline { 2 - 2 } & Medial $4.4 \pm 3.24 \mathrm{~mm}(\mathrm{n}=8)$ \\
\hline $\begin{array}{l}\text { Descending the } \\
\text { greater trochanter }\end{array}$ & $76.48 \pm 17.85 \mathrm{~mm}$ \\
\hline $\begin{array}{l}\text { Introduction of the } \\
\text { stem into the distal part }\end{array}$ & $81.77 \pm 25.7 \mathrm{~mm}$ \\
\hline $\begin{array}{l}\text { Mean value of femur } \\
\text { resection }\end{array}$ & $57.63 \pm 16.39 \mathrm{~mm}$ \\
\hline
\end{tabular}

Pain in the hip joint according to the VAS scale before surgery was $6.9 \pm 1.6$. At the follow-up examination, it was $3.4 \pm 1.1$. In the lumbar spine, the pain score was $6.1 \pm 2.1$ before the operation and $2.1 \pm 2.2$ in the postoperative period. It is worth noting that pain in the LS prevailed over pain in the hip joint in $44.7 \%$ of cases. It was more often observed in patients with bilateral dysplasia. The mean Harris score was $48.9 \pm 19.3$ before surgery, and 55.6 \pm 17.2 at the follow-up.

Pain relief in the lumbar spine may be associated with changes in the lateral radiographs. Before surgery, this group of patients had excessive anteversion of the pelvis which led to hyperlordosis and excessive tilt of the sacrum. In the postoperative period, pelvic anteversion returned to normal, like the rest of the angles of the lumbar-pelvic balance, which was reflected in a decrease in pain in the spine after surgery. Table 2 shows changes in the spinal-pelvic relationship.

Table 2

Changes in the spinal-pelvic relationship before and after surgery

\begin{tabular}{|l|c|c|}
\hline \multicolumn{1}{|c|}{ Parameter } & $\begin{array}{c}\text { Mean value } \\
\text { before surgery }\end{array}$ & $\begin{array}{c}\text { Mean value after } \\
\text { surgery }\end{array}$ \\
\hline SS, degrees & $46.4 \pm 9.9$ & $39.0 \pm 10.9$ \\
\hline PT, degrees & $-23.9 \pm 35.0$ & $6.5 \pm 3.4$ \\
\hline GLL, degrees & $64.1 \pm 13.5$ & $55.2 \pm 7.3$ \\
\hline
\end{tabular}

In the early postoperative period, 13 complications were noted in 12 patients: three sciatic nerve neuropathies (two of them resolved in the late period); 9 cases of dislocation of the femoral component; two cases of fracture of the proximal femur fragment that were synthesized by cerclage sutures; one case of distal fragment fracture which required a MOS plate. One case of early loosening of the femoral component after a follow-up of four years required revision with exchange of the femoral component (Fig. 4).

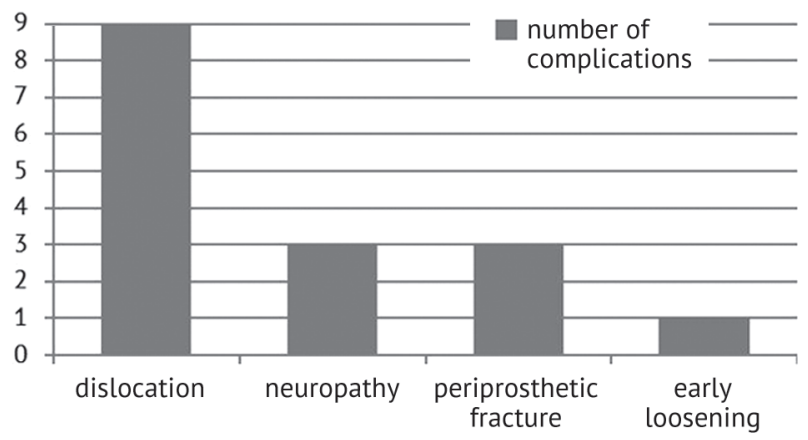

Fig. 4 Complications in patients with Crowe type IV hip dysplasia, by terms in years

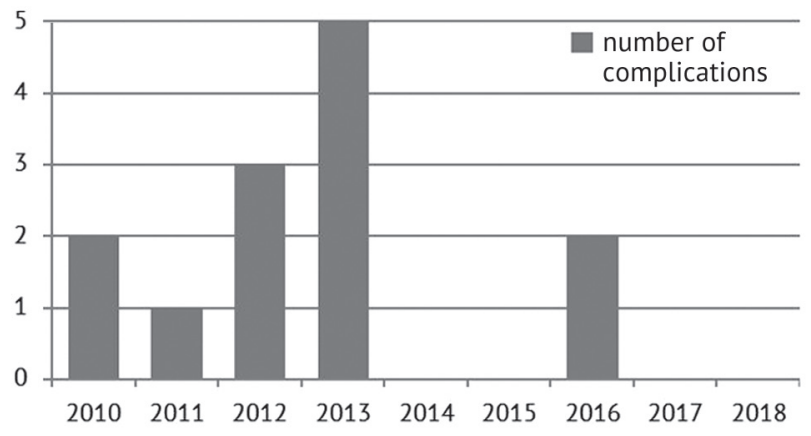

Fig. 5 Complications in patients with DDH according to Crowe grade IV by years

Additionally, annual complications in the group were analyzed. The diagram shows that the largest number of complications was up to year 2013, inclusive. Two complications were noted in 2016 in a 57-year-old patient, a combination of a periprosthetic fracture and hip dislocation. This type of surgical treatment was performed by two surgeons of orthopaedic department No. 2. The decrease in the number of complications has been associated with the development of the method.

\section{DISCUSSION}

Hip arthroplasty in congenital hip dislocation is a complex multistep operation, one of the stages of which is a shortening subtrochanteric osteotomy. The preference in choosing this technique is based on the fact that the proximal femur with the greater and lesser trochanters is restored maintaining the fixation of the muscles to the greater trochanter upon fusion of the proximal and distal fragments. Thus, good function of the prosthetic joint is expected with the reconstructed anatomy.
The most frequent complication in our group was dislocation of the femoral component of the implant (69 \% of all complications, $n=9$ ). A number of articles report that the most frequent complication is intraoperative fracture during the insertion of the femoral component (5-22 \%) [10, 11]. In a study by Rollo et al. [12], only one intraoperative fracture was recorded (6\%). All osteotomies healed in an average of 12 weeks (range, 10 to 15 ) which corresponds to the average time for consolidation 
of the femur. These results are consistent with those reported in other studies using the same osteotomy techniques [13]. Sciatic nerve neuropathy is reported in 0.8 to $13 \%$ of patients who underwent such hip arthroplasty $[14,15]$. In our study, the incidence of this complication was $5 \%(n=3)$, or $23 \%$ of the total number of complications. Leg lengthening of more than $4 \mathrm{~cm}$ is considered to be an indication for a shortening osteotomy of the femur to prevent sciatic nerve neuropathy. However, Eggli et al. [16] found no association between leg lengthening and neuropathy in 508 patients who underwent hip arthroplasty.

A number of authors found the relationship between clinical results after hip arthroplasty with changes in the spinal-pelvic relationship that improved for the better and reduced pain without affecting the spine $[17,18]$.

In a study by Rollo et al. [12] the mean preoperative leg length discrepancy was $45 \mathrm{~mm}$ (range, 38-70 mm) and decreased to $12 \mathrm{~mm}$ (range, 9-1.6) after surgery for unilateral lesions.

The simplest type for planning resection is a bilateral unsupportive dislocation, untreated in childhood. At the first stage, shortening is carried out to the extent that it is sufficient to reproduce the normal anatomy of the hip joint, with the restoration of the centre of rotation without tensioning the sciatic nerve. At the second stage, it is enough to reproduce the amount resected, achieving the minimum difference in the length of the limbs and placing the axes of rotation of the knee joints at the same level. The limb length at an intermediate stage was compensated with orthopedic shoes.

The development of the method of femur shortening has led to the emergence of various modifications of the proximal shortening, including variants of intertrochanteric and distal shortening [19] of the femur. The variety of proposed options for osteotomies (Chevron, oblique, transverse, Z-shaped) reflect the search for the most effective solution of the problem of maximum adaptation of bone fragments to each other and an increase in the area of contact, which ensures bone fusion of the fragments in a short time. Shortening of the femur may be achieved by proximal or distal osteotomy. In Paavilainen's osteotomy, the femoral metaphysis is resected, which is a potential risk of pseudarthrosis in the region of the greater trochanter [20]. In our opinion, shortening subtrochanteric osteotomy is a safer option with better results. Moreover, the metaphysis of the femur remains preserved in this technology.

\section{CONCLUSION}

Hip joint arthroplasty for type IV dysplasia (Crowe) is an operation of high complexity and is associated with a high risk of complications (19.7 \%). The most common complication is dislocation of the femoral component, amounting to $69.2 \%$ of the total number of complications, while this operation is reproducible.

Pelvic components of the minimum size (90.9\%) up to $46 \mathrm{~mm}$ inclusive with the use of additional screw fixation in $60.6 \%$ of cases is a need for arthroplasty performance in this category of patients, and the use of a pelvic component of $42 \mathrm{~mm}$ in diameter may be required.

Shortening subtrochanteric osteotomy in hip arthroplasty for patients with type IV of dysplasia provides restoration of hip biomechanics with a slight displacement of the rotation centre up to $10 \mathrm{~mm}$ and normalizes the spinal-pelvic relationship.

\section{REFERENCE}

1. Crowe J.F., Mani V.J., Ranawat C.S. Total hip replacement in congenital dislocation and dysplasia of the hip. J. Bone Joint Surg. Am., 1979 , vol. 61 , no. 1 , pp. 15-23.

2. Hartofilakidis G., Stamos K., Ioannidis T.T. Low friction arthroplasty for old untreated congenital dislocation of the hip. J. Bone Joint Surg. Br., 1988, vol.70, no. 2, pp. 182-186.

3. Eftekhar N.S. Congenital dysplasia and dislocation in total hip arthroplasty. St. Louis, Mosby. 1993, pp. 925-963.

4. Gustke K. The dysplastic hip: not for the shallow surgeon. Bone Joint J., 2013, vol.95-B, no. 11 Suppl. A, pp. 31-36. DOI: 10.130 2/0301-620X.95B11.32899.

5. Klisic P., Jankovic L. Combined procedure of open reduction and shortening of the femur in treatment of congenital dislocation of the hips in older children. Clin. Orthop. Relat. Res., 1976, no. 119, pp. 60-69.

6. Sponseller P.D., McBeath A.A. Subtrochanteric osteotomy with intramedullary fixation for arthroplasty of the dysplastic hip. A case report. J. Arthroplasty, 1988, vol. 3, no. 4, pp. 351-354. DOI: 10.1016/s0883-5403(88)80036-6.

7. Cabanela M.E. Total hip arthroplasty for developmental dysplasia of the hip. Orthopedics, 2001, vol. 24, no. 9, pp. 865-866.

8. Park Y.S., Moon Y.W., Lim S.J. Revision total hip arthroplasty using a fluted and tapered modular distal fixation stem with and without extended trochanteric osteotomy. J. Arthroplasty, 2007, vol. 22, no. 7, pp. 993-999. DOI: 10.1016/j.arth.2007.03.017.

9. Krych A.J., Pagnano M.W. CAOS: Greater Precision, Doubtful Clinical Benefit-Affirms. Seminars in Arthroplasty, 2009, vol. 20, no. 1 , pp. 58-59.

10.Krych A.J., Howard J.L., Trousdale R.T., Cabanela M.E., Berry D.J. Total hip arthroplasty with shortening subtrochanteric osteotomy in Crowe type-IV developmental dysplasia: surgical technique. J. Bone Joint Surg. Am., 2010, vol. 92, no. Suppl. 1, Pt. 2, pp. 176187. DOI: 10.2106/JBJS.J.00061.

11.Reikerås O., Haaland J.E., Lereim P. Femoral shortening in total hip arthroplasty for high developmental dysplasia of the hip. Clin. Orthop. Relat. Res., 2010, vol. 468, no. 7, pp. 1949-1955. DOI: 10.1007/s11999-009-1218-7. 
12.Rollo G., Solarino G., Vicenti G., Picca G., Carrozzo M., Moretti B. Subtrochanteric femoral shortening osteotomy combined with cementless total hip replacement for Crowe type IV developmental dysplasia: a retrospective study. J. Orthop. Traumatol., 2017, vol.18, no. 4, pp. 407-413. DOI: 10.1007/s10195-017-0466-7.

13.Takao M., Ohzono K., Nishii T., Miki H., Nakamura N., Sugano N. Cementless modular total hip arthroplasty with subtrochanteric shortening osteotomy for hips with developmental dysplasia. J. Bone Joint Surg. Am., 2011, vol. 93, no. 6, pp. 548-555. DOI: 10.2106/ JBJS.I.01619.

14.Hasegawa Y., Iwase T., Kanoh T., Seki T., Matsuoka A. Total hip arthroplasty for Crowe type IV developmental dysplasia. J. Arthroplasty, 2012, vol. 27, no. 9, pp. 1629-1635. DOI: 10.1016/j.arth.2012.02.026.

15.Lai K.A., Shen W.J., Huang L.W., Chen M.Y. Cementless total hip arthroplasty and limb-length equalization in patients with unilateral Crowe type-IV hip dislocation. J. Bone Joint Surg. Am., 2005, vol. 87, no. 2, pp. 339-345. DOI: 10.2106/JBJS.D.02097.

16.Eggli S., Hankemayer S., Müller M. Nerve palsy after leg lengthening in total replacement arthroplasty for developmental dysplasia of the hip. J. Bone Joint Surg. Br., 1999, vol. 81, no. 5, pp. 843-845. DOI: 10.1302/0301-620x.81b5.9610.

17.Weng W., Wu H., Wu M., Zhu Y., Qiu Y., Wang W. The effect of total hip arthroplasty on sagittal spinal-pelvic-leg alignment and low back pain in patients with severe hip osteoarthritis. Eur. Spine J., 2016, vol. 25, no. 11, pp. 3608-3614. DOI: 10.1007/s00586016-4444-1.

18.Ben-Galim P., Ben-Galim T., Rand N., Haim A., Hipp J., Dekel S., Floman Y. Hip-spine syndrome: the effect of total hip replacement surgery on low back pain in severe osteoarthritis of the hip. Spine, 2007, vol. 32, no. 19, pp. 2099-2102. DOI: 10.1097/ BRS.0b013e318145a3c5.

19.Guo C.Y., Liang B.W., Sha M., Kang L.Q., Wang J.Z., Ding Z.Q. Cementless arthroplasty with a distal femoral shortening for the treatment of Crowe type IV developmental hip dysplasia. Indian J. Orthop., 2015, vol. 49, no. 4, pp. 442-446. DOI: 10.4103/00195413.159652.

20.Tikhilov R.M., Mazurenko A.V., Shubniakov I.I., Denisov A.O., Blizniukov V.V., Bilyk S.S. Rezultaty endoprotezirovaniia tazobedrennogo sustava s ukorachivaiushchei osteotomiei po metodike T. Paavilainen pri polnom vyvikhe bedra [Results of the hip arthroplasty with shortening osteotomy according to T. Paavilainen technique for total dislocation of the hip]. Travmatologiia $i$ Ortopediia Rossii, 2014, no. 1 (71), pp. 5-15. (in Russian)

Received: 20.11.2019

\section{Information about the authors:}

1. Lev S. Shnaider, M.D., Novosibirsk Research Institute Of Traumatology And Orthopaedics N.a. Ya.l. Tsivyan, Novosibirsk, Russian Federation, Email: Leonsch@mail.ru

2. Oleg I. Golenkov, M.D., Novosibirsk Research Institute Of Traumatology And Orthopaedics N.a. Ya.l. Tsivyan, Novosibirsk, Russian Federation

3. E.U. Turgunov, M.D., Novosibirsk Research Institute Of Traumatology And Orthopaedics N.a. Ya.l. Tsivyan, Novosibirsk, Russian Federation 4. Maksim V. Efimenko, M.D., Novosibirsk Research Institute Of Traumatology And Orthopaedics N.a. Ya.l. Tsivyan, Novosibirsk, Russian Federation 5. M.A. Stepankova, M.D.,

Novosibirsk Research Institute Of Traumatology And Orthopaedics N.a. Ya.l. Tsivyan, Novosibirsk, Russian Federation 6. Vitaliy V. Pavlov, M.D., Ph.D.,

Novosibirsk Research Institute Of Traumatology And Orthopaedics N.a. Ya.l. Tsivyan, Novosibirsk, Russian Federation 